\title{
Steep unidirectional wave groups - fully nonlinear simulations vs. experiments
}

\author{
L. Shemer and B. K. Ee \\ School of Mechanical Engineering, Tel Aviv University, Tel Aviv 69978, Israel \\ Correspondence to: L. Shemer (shemer@eng.tau.ac.il)
}

Received: 1 July 2015 - Published in Nonlin. Processes Geophys. Discuss.: 24 July 2015

Revised: 18 November 2015 - Accepted: 26 November 2015 - Published: 4 December 2015

\begin{abstract}
A detailed quantitative comparison of fully nonlinear computations with the measurements of unidirectional wave groups is presented. Computational results on evolving wave groups were compared with previous available experiments. The local surface elevation variation, the evolution of envelope shapes, the velocity of propagation of the steepest crests in the group and their relation to the height of the crests were obtained numerically and experimentally. Conditions corresponding to incipient wave breaking were investigated in greater detail. The results shed additional light on mechanisms leading to the breaking of steep waves, as well as on the crucial importance of exact matching between initial conditions in computations and experiments.
\end{abstract}

\section{Introduction}

Over the past few decades, rogue, or freak, waves have attracted considerable interest due to their destructive impact on offshore structures and ships (Dysthe et al., 2008; Kharif et al., 2009). A number of possible mechanisms for rogue wave generation have been explored. Wave-current and wave-bathymetry interactions may result in appearance of rogue waves (Kharif et al., 2009). Extremely steep waves in the ocean are thus usually affected by the directional characteristics of the wave field. Nevertheless, considerable effort has been invested in recent decades to study unidirectional wave fields. The accumulated results clearly demonstrate that investigation of both deterministic and random unidirectional waves can lead to a better understanding of mechanisms leading to appearance of rogue waves in the presence of directional spreading as well. Experimental studies of 2-D wave fields in wave basins require large and expensive facilities and are subject to numerous limitations on the wave parameters. Generation of unidirectional wave groups in long tanks by a computer-controlled wavemaker offers significant advantages in terms of availability and versatility of operational conditions. Extremely steep waves can be generated due to constructive interference of numerous harmonics. While this focusing mechanism is basically linear, it is strongly affected by nonlinearity (Shemer et al., 2007; Bateman et al., 2012). An alternative, essentially nonlinear, mechanism is related to the specific properties of the governing equations. To that end, the nonlinear Schrödinger (NLS) equation (Zakharov, 1968; Hasimoto and Ono, 1972), applicable for description of diverse nonlinear physical phenomena, is the simplest theoretical model describing the evolution of narrowbanded wave groups in deep and intermediate depth water. This equation attracts special interest in rogue waves studies since it admits analytical solutions such as the socalled Kuznetsov-Ma breathing solitons (Kuznetsov, 1977; Ma, 1979). These solutions of the NLS equation present spatially localized patterns that oscillate in time. The closely related Akhmediev breather (Akhmediev et al., 1987) is periodic in space. When the periodicity in time and space tends to infinity, both these types of solution tend to a simple Peregrine breather (PB) (Peregrine, 1983). It is localized in time and space, breathes only once and attains a maximum crest height that exceeds that of the background wave train by a factor of 3. For this reason, the Peregrine and other breathertype solutions of the NLS equation have been proposed as rogue wave prototypes (Dysthe and Trulsen, 1999; Osborne et al., 2000; Shrira and Geogjaev, 2010).

Shemer and Alperovich (2013) conducted a series of experiments on the evolution of the Peregrine breather (PB) along a wave tank. They demonstrated that the experimental 
results diverge from Peregrine's solution of the NLS equation. Notable asymmetry of the crest was observed, in agreement with many earlier studies of extremely steep waves (Babanin et al., 2007). The discrepancy between the fully symmetric NLS solution and experiments manifests itself mainly in significant asymmetric widening of the wave spectrum, as well as in notably slower amplification of the wave height than predicted by the PB solution. Moreover, these experiments suggested that, contrary to the behavior of the PB, there would be no return to the initial nearly monochromatic wave train. Similar conclusions based on fully nonlinear simulations of PB evolution in time were reached by Slunyaev and Shrira (2013). Shemer and Alperovich (2013) demonstrated that the modified nonlinear Schrödinger (MNLS, or Dysthe) (Dysthe, 1979) equation was advantageous in describing the PB evolution along the laboratory tank as compared to the NLS equation. The improved performance of the Dysthe model was attributed in Shemer and Alperovich (2013) to the additional fourth-order terms in this equation that account for the finite spectral width (Kit and Shemer, 2002). It was thus demonstrated that the statement made by Chabchoub et al. $(2011,2012)$ and elsewhere that PB has been observed in water wave experiments is essentially unsubstantiated. Shemer $(2015,2016)$ demonstrated that the spatial evolution patten of PB does not differ from that of any initially narrow-banded modulated wave train.

Shemer and Liberzon (2014) took advantage of the fact that the spectral widening, being an essentially nonlinear process, occurs at slow spatial and temporal scales. Hence, it was found that the wave train behavior with background steepness of about 0.1 is still described by the PB solution of the NLS equation with reasonable accuracy, as long as the surface elevation spectrum remains sufficiently narrow and the maximum wave height in the train remained below approximately twice that of the background. This observation enabled Shemer and Liberzon (2014) to utilize the available PB analytic solution to design experiments with PB in which the height of the steepest wave in the train at a prescribed measuring location can be controlled, thus facilitating quantitative studies of the incipient wave breaking. Their study was motivated by an earlier attempt by Shemer (2013) to examine the kinematics of the steep wave on the verge of breaking using the Zakharov equation (Zakharov, 1968). By comparing the computational results with experimental observations reported in Shemer et al. (2007), the conclusion was reached that wave breaking may occur when the horizontal liquid velocity at the crest becomes sufficiently high (Shemer, 2013). These computations also showed that the maximum negative vertical Lagrangian acceleration seems to remain significantly below the acceleration of gravity $g$, so that the Phillips dynamic breaking criterion cannot be satisfied (Phillips, 1958). Computations of steep wave kinematics accurate up to the third order in the wave steepness demonstrated, though, that this approximation, while largely adequate for determination of the shape of the surface eleva- tion, was insufficient for the accurate characterization of the kinematics of steep waves (Shemer, 2013). In order to overcome this limitation, the kinematic parameters of the steepest wave in the PB-like wave train were determined experimentally in Shemer and Liberzon (2014) simultaneously with estimates of the propagation velocity of the steepest crest. To this end, two synchronized video cameras were used to image the wave field. The maximum possible horizontal Lagrangian velocities and accelerations at the surface of steep water waves were measured by particle tracking velocimetry (PTV) for gradually increasing crest heights, up to the inception of a spilling breaker. Actual crest and phase velocities were estimated from video-recorded sequences of the instantaneous wave shape as well as from surface elevation measurements by wave gauges. The slowdown of the crest as it grows steeper was observed. It was suggested in Shemer and Liberzon (2014) that the inception of a spilling breaker is associated with the horizontal velocity of water particles at the crest attaining that of the crest, thus confirming the kinematic criterion for the inception of breaking.

In the present study, we aim to extend the numerical analysis of the conditions prevailing at the inception of breaking of the steepest crest in the PB-like wave train by carrying out fully nonlinear simulations. The simulations were performed using the conformal mapping method approach developed by Chalikov and Sheinin $(1998,2005)$ (hereafter referred to as the CS numerical scheme). A somewhat different implementation of this approach was suggested by Milewski et al. (2010). Recently, Perić et al. (2014) reported on direct numerical simulations based on the volume of fluid method to solve the two-phase Navier-Stokes equations. In their study, Peregrine breather dynamics was investigated up to the initial stages of wave breaking.

In Sect. 2, the difference between the spatial and temporal evolution of the wave field is discussed. In Sect. 3, we give details about the solver that is based on the conformal mapping method and stress that the resulting numerical solution describes the temporal evolution starting from an initial spatial distribution. In Sect. 4, the computational results are discussed for both the temporal evolution problem and then for the spatial evolution case. The corresponding experimental results are also presented and compared directly with the numerical simulations. In Sect. 5, the numerical and experimental results are discussed and the conclusions are drawn.

\section{Wave parameters: temporal vs. spatial evolution cases}

The CS numerical scheme enables computation of a unidirectional wave field in time, starting from a given spatial distribution as an initial condition, under the assumption of potential flow. In laboratory experiments, however, waves are generated by a wavemaker usually placed at one end of the experimental facility. The experimental data are com- 
monly accumulated only at preselected fixed locations within the tank where the sensors are placed. Quantitative comparison of numerical computations with experimental results gained in those experiments may thus constitute a complicated task. For a narrow-banded wave field this problem has been considered by Shemer and Dorfman (2008). For such wave fields, the envelope equations like the NLS and Dysthe models often provide adequate results. In the narrow-banded models the temporal $t$ and spatial $x$ coordinates are related by the group propagation velocity, $c_{\mathrm{g}}$, thus enabling modification of the governing temporal evolution equations to a spatial form. The spatial form of the Dysthe model was presented by Lo and Mei (1985). Numerical computations based on the Dysthe model for unidirectional wave groups propagating in a long wave tank indeed provided good agreement with experiments (Shemer et al., 2002). The spatial version of the Dysthe equation was also derived from the spatial form of the Zakharov equation (Shemer et al., 2001, 2007) that is free of any restrictions on the spectrum width (Kit and Shemer, 2002).

As demonstrated in Shemer and Dorfman (2008), the availability of the spatial form of the evolution model is insufficient to pose the initial conditions for the numerical simulations that correspond exactly to those in experiments. In the present work, the temporal evolution is computed by a fully nonlinear solver of the 2-D potential equations in finite water depth. Following earlier works (Shemer and Alperovich, 2013; Shemer and Liberzon, 2014), the solution of the spatial version of the NLS equation is used to set the initial conditions. For a narrow-banded deep water wave group, the spatial and temporal variations of the surface elevation $z$ at the leading order can be presented as

$\zeta(x, t)=\operatorname{Re}\left[a(x, t) \cdot e^{i\left(k_{0} x-\omega_{0} t\right)}\right]$.

Here the radian frequency $\omega_{0}=2 \pi / T_{0}, T_{0}$ being the carrier wave period, and the wavenumber $k_{0}=2 \pi / \lambda_{0}, \lambda_{0}$ being the carrier wavelength, satisfy the finite water depth dispersion relation $\omega_{0}^{2}=g k_{0} \tanh \left(k_{0} h\right)$. In Eq. (1), $a$ is the slowly varying complex group envelope. The wave steepness is defined as $\epsilon=a_{0} k_{0}$, where $a_{0}$ is the characteristic wave amplitude. The wave train given by Eq. (1) propagates with the group velocity $c_{\mathrm{g}}=\left(\frac{\partial \omega}{\partial k}\right)_{k=k_{0}}$. Following Mei (1989) and Shemer et al. (1998), in intermediate water depth the spatial NLS equation for the complex normalized envelope $Q=a(x, t) / a_{0}$ is given by

$-i Q_{X}+\alpha Q_{T T}+\beta|Q|^{2} Q=0$,

where the scaled dimensionless temporal and spatial coordinates are $T=\epsilon \omega_{0}\left(x / c_{\mathrm{g}}-t\right)$ and $X=\epsilon^{2} k_{0} x$, respectively. The coefficients in the NLS equation have the following di- mensionless form:

$$
\begin{aligned}
\alpha & =-\frac{\omega_{0}^{2}}{2 k_{0} c_{\mathrm{g}}{ }^{3}} \frac{\partial c_{\mathrm{g}}}{\partial k}, \\
\beta & =\frac{1}{n}\left[\frac{\cos h\left(4 k_{0} h\right)+8-2 \tanh ^{2}\left(k_{0} h\right)}{16 \sin h^{4}\left(k_{0} h\right)}\right. \\
- & \left.\frac{1}{2 \sin h^{2}\left(2 k_{0} h\right)} \frac{\left(2 \cos h^{2}\left(k_{0} h\right)+n^{2}\right)}{\frac{k_{0} h}{\tanh \left(k_{0} h\right)}-n^{2}}\right],
\end{aligned}
$$

where the parameter $n=c_{\mathrm{g}} / c_{\mathrm{p}}$ represents the ratio of group and phase velocities and is given by

$n=\frac{1}{2}\left\{1+\frac{2 k_{0} h}{\sin h\left(2 k_{0} h\right)}\right\}$.

For the deep water case, $k_{0} h \rightarrow \infty$ and $\alpha=\beta=1$. The Peregrine breather solution of the NLS equation for intermediate water depth (Eq. 2) is

$$
Q=-\sqrt{2 \frac{\alpha}{\beta}} e^{-2 i \alpha X}\left[1-\frac{4(1-4 i \alpha X)}{1+4 T^{2}+16(\alpha X)^{2}}\right] .
$$

Equations (1) and (6) provide variation of the instantaneous surface elevation envelope in time and in space, with focusing corresponding to $T=X=0$. In Shemer and Alperovich (2013) and Shemer and Liberzon (2014), the wavemaker driving signal as a function of time was chosen using the deep-water version of Eq. (2) and the prescribed focusing distance from the wavemaker, $x_{0}$, by substituting $X=X_{0}=-\epsilon^{2} k_{0} x_{0}$ into Eq. (6). The relative height of the initial hump in the wave amplitude distribution at the wavemaker in Shemer and Liberzon (2014) exceeded $10 \%$ above the background.

The present study has been carried out in the $18 \mathrm{~m}$ long, $1.2 \mathrm{~m}$ wide and $0.9 \mathrm{~m}$ deep wave tank (water depth $h=0.6 \mathrm{~m}$ ). More details about the experimental facility are given in Shemer et al. (1998). The carrier wave period $T_{0}=$ $0.8 \mathrm{~s}$ was selected, corresponding to the carrier wavelength $\lambda_{0}=1.0 \mathrm{~m}$ and the dimensionless water depth $k_{0} h=3.77$. For these parameters, both coefficients in the NLS equation (Eq. 2) given by Eqs. (3) and (4) in fact differ from unity: $\alpha=1.078$ and $\beta=0.711$. The carrier wave amplitude of $\zeta_{0}=\sqrt{\frac{2 \alpha}{\beta}} a_{0}=0.026 \mathrm{~m}$ was used, corresponding to the nonlinearity $\epsilon=k_{0} a_{0}=0.094$.

Since the nonlinear numerical solver in the present study requires an initial condition as a given spatial distribution at a certain instant, the following procedure to determine the appropriate initial spatial distribution was adopted.

Using Eq. (6), a value of $x_{0}$ is specified at which the prescribed maximum crest of the PB is to be located. Note that in physical terms, this initial condition corresponds to a situation in which the whole wave train is placed upstream of the wavemaker. Due to the focusing properties of the NLS equation in sufficiently deep water $\left(k_{0} h>1.36\right)$, the maximum wave height in the train increases in the course of the 
evolution. In Shemer and Liberzon (2014), the amplification at the wavemaker was about $20 \%$. To obtain a similar evolution pattern in the present study, the initial height of the PB hump has to be significantly smaller than that. The amplification corresponding to $5 \%$ was selected. The temporal variation of the surface elevation at $x=x_{0}, \zeta\left(x_{0}, t\right)$, can be calculated using $T\left(x_{0}, t\right)$ and $X\left(x_{0}\right)$. The maximum amplification occurs at $T=0$ corresponding to the dimensional time $t_{\max }=-x_{0} / c_{\mathrm{g}}$; the resulting $\zeta(t)$ is symmetric with respect to $t_{\max }$. Note that at the instant $t=t_{\max }$, the spatial distribution $\zeta\left(x, t_{\max }\right)$ is not fully symmetric with respect to $x_{0}$ due to the presence of term $e^{-2 i \alpha X}$ in Eq. (6). It should be stressed that in experiments as well as in numerical simulations, the actual extent of the wave group is necessarily finite. In the experiments of Shemer and Alperovich (2013) and Shemer and Liberzon (2014), the wave train with a duration of 70 carrier wave periods was generated by the wavemaker. The duration of the wave train of $70 T_{0}$ is long enough to eliminate the effect of truncation onto the central part of the train where the hump is located and which is of particular interest, and on the other hand sufficiently short to prevent contamination of the measured surface elevation by possible reflection from the far end of the tank. In the spatial domain, this duration of the wave train corresponds to $35 \lambda_{0}$. The numerical method applied in the present study assumes spatially periodic boundary conditions. Since the initial spatial distribution is not periodic, the periodicity was enforced by applying a linear tapering window over wavelength $2 \lambda_{0}$ at the edges of the wave train. As a result, the effective, undisturbed by tapering, wave group extended initially for about $32 \lambda_{0}$. To allow evolution of the wave train unaffected by boundaries, the computational domain was selected as $\left[-32 \lambda_{0}, 32 \lambda_{0}\right]$, with $x=0$ corresponding to the location of the maximum crest at the initial instant of the computations, $t=0$. The temporal initial condition adopted in the study is plotted in the top panel of Fig. 1. The corresponding spatial variation of the surface elevation with the same maximum crest height is plotted in the bottom panel of the same figure.

\section{Numerical solution methodology}

The temporal evolution of the initial wave field presented in the bottom panel of Fig. 1 is obtained by solving potential flow equations following the fully nonlinear numerical approach developed by Chalikov and Sheinin $(1998,2005)$. The CS numerical scheme is known to be robust and does not have limitations in terms of wave steepness. It has been extensively and successfully used for numerical simulations of numerous problems related to evolution of nonlinear waves. The conformal mapping method is applied to solve Laplace's equation for the velocity potential. Surface tension effects are neglected. The principal equations are re-written in a surfacefollowing coordinate system ( $\xi$ and $\zeta$ ) and reduced to two time-evolutionary equations for the surface elevation $z$ and
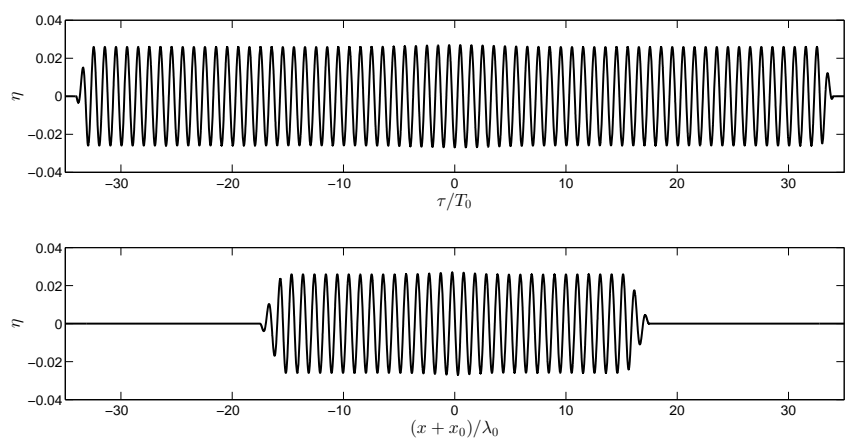

Figure 1. The prescribed initial variation of the surface elevation $\eta$ in the temporal (top) and spatial (bottom) domains for the carrier wave period $T_{0}=0.8 \mathrm{~s}$ and background carrier amplitude $\zeta_{0}=0.026 \mathrm{~m}$; calculated for $X=-2.613\left(x_{0}=31 \mathrm{~m}\right)$ in Eq. (6).

velocity potential $\phi^{\mathrm{s}}$ at the surface. The evolutionary equations representing the kinematic and dynamic boundary conditions at the free surface are written in terms of the Fourier coefficients of $z$ and $\phi^{\mathrm{s}}$. This enables the reduction of the evolutionary equations into a system of time-dependent ordinary differential equations for $4 M+2$ Fourier coefficients of $z$ and $\phi^{\mathrm{s}}$, coupled with appropriate initial conditions. Here $M$ refers to the truncation number of the Fourier series.

The initial surface elevation is given as a function of the physical variable $x$. To solve the problem in the mapped space, this initial condition has to be converted into a function of equally spaced $\xi$. This is done by an iteration procedure.

For time integration, a fourth-order Runge-Kutta scheme was used. We refer the reader to Chalikov and Sheinin (2005) for further details. In the present computations, the dimensional spatial discretization interval was $\lambda_{0} / 256$, so that the total number of spatial points $N=17920$; the truncation number for the Fourier series is $M=N / 9$. This value of $M$ effectively means that waves with wavelengths shorter than $1.5 \mathrm{~cm}$ where capillary effects become dominant are disregarded. The dimensional integration step in time is $\mathrm{d} t=$ $3.125 \times 10^{-6} \mathrm{~s}$.

The CS numerical scheme allows for the computation of the velocity potential as a function of two parameters: $\xi$ and $\zeta$; the velocity potential for the entire domain can thus be calculated at any instant. In view of the focus of the present study, the output parameters of the numerical integration are the surface elevation $z$, velocity potential at the surface $\phi^{\mathrm{s}}$ and the physical spatial coordinate $x$, which are all functions of $\xi$ and $t$. In order to record the data for future use, the results for surface elevation, the coordinates $x$ and nondimensional velocity potential are saved at every $3.125 \mathrm{~ms}$. Note also that the spline interpolation procedure is needed to obtain values of the surface elevation $z$ and the velocity potential $\phi^{\mathrm{s}}$ at equally spaced values of $x$.

Milewski et al. (2010) have also employed the conformal mapping method to investigate the unsteady evolution of 2-D 
fully nonlinear free surface gravity-capillary solitary waves for infinite depth. Though their numerical approach is similar to that of CS, certain differences between the methods exist. The numerical approach of Milewski et al. (2010) was implemented in our computations as well. No significant differences with the results based on the CS numerical scheme were obtained, thus further demonstrating the robustness of the present results.

\section{Numerical and experimental results}

In Fig. 2, the spatial instantaneous wave surface profile is plotted for several characteristic selected instants. As mentioned above, the origin of the frame of references $x=0$ corresponds to the location of the maximum crest in the initial spatial distribution. The simulations demonstrate that abnormally high waves appear at both edges of the wave train as a result of truncation and tapering of the infinite wave train defined by Eqs. (1) and (6) as specified in the previous section. A similar phenomenon was observed in experiments with truncated wave trains reported in earlier works (Shemer and Alperovich, 2013; Shemer and Liberzon, 2014). The effect of truncation, however, apparently does not extend to the central part of the wave train even at relatively long times, as can be seen from the upper curves in this figure. The effect of nonlinear focusing on the behavior of this central part of the train in the vicinity of the hump is of principal interest in this study. The dashed lines in Fig. 2 originate at the leading edge, the center and the trailing edge of the initial wave train and correspond to the location of the point propagating with the group velocity $c_{\mathrm{g}}=0.63 \mathrm{~m} \mathrm{~s}^{-1}$. It transpires from the figure that the leading edge of the train indeed propagates with the speed that is close to $c_{\mathrm{g}}$, while the trailing edge seems to move somewhat faster. The propagation velocity of the steepest crest, however, exceeds notably the group velocity $c_{\mathrm{g}}$, in agreement with the experimental observations and the numerical simulation based on the Dysthe equation in Shemer and Alperovich (2013).

The spatial variation of the velocity of the fluid at the surface $u_{\mathrm{h}}=\partial \phi^{\mathrm{s}} / \partial x$ is plotted in Fig. 3 at the same instants as in Fig. 2, but in a frame of reference that moves with a group velocity $c_{\mathrm{g}}$. Only the central part of the wave train is shown. The horizontal fluid velocity at the steepest crest increases notably during the focusing process. At the upper curve in Fig. 3, the fast increase in the horizontal velocity at the crest is clearly seen. Note that in earlier experiments by Shemer and Liberzon (2014), wave breaking was indeed observed at comparable distances from the wavemaker. The individual waves in Fig. 3 manifest variable left-right asymmetry.

Measurements in a wave tank are routinely performed using wave gauges spread along the facility. To facilitate the direct comparison between numerical and experimental results, we need to first determine the location of the wavemaker in our numerical simulations. Then, we examine "ver-

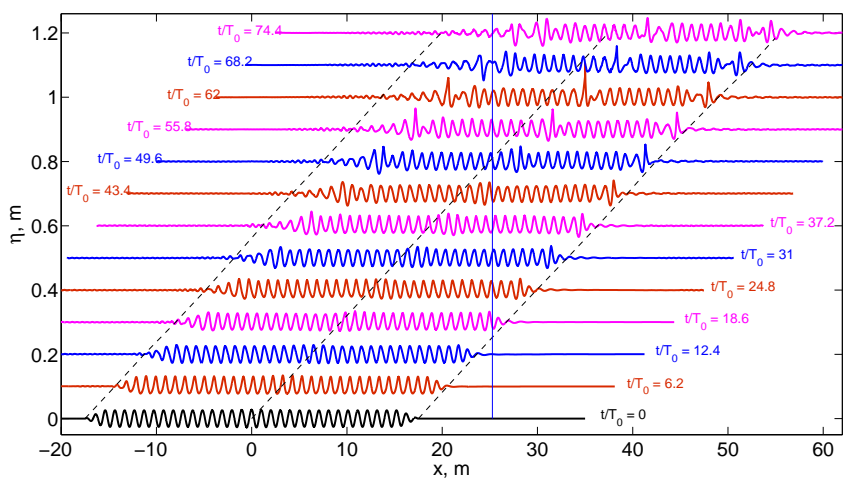

Figure 2. The temporal evolution of the surface elevation $\eta$ in a fixed reference frame; wave parameters as in Fig. 1. The vertical line marks the location of the wavemaker at $x=x_{\mathrm{wm}}=25.273 \mathrm{~m}$; broken lines correspond to propagation with the group velocity $c_{\mathrm{g}}$.

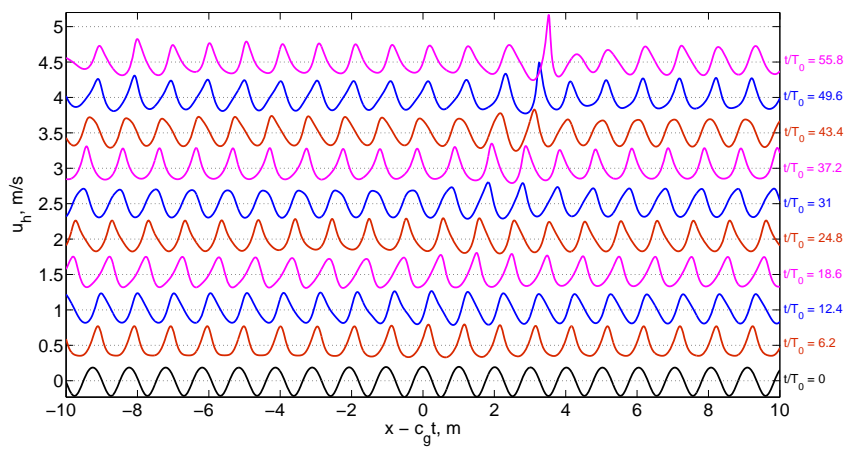

Figure 3. The temporal evolution of the horizontal fluid velocity in a moving reference frame; wave parameters as in Fig. 1.

tical" cross sections of the data as presented in Fig. 2 at fixed locations relative to the adopted coordinate of the wavemaker. The location of the wavemaker is identified by the vertical line in the latter figure where $x=x_{\mathrm{wm}}=25.273 \mathrm{~m}$. The temporal variation of the surface elevation is plotted in Fig. 4 at selected locations that cover the range of the wave gauge positions in the experiment, starting with that at $x_{\mathrm{wm}}$ or $x_{*}=0$, the variable $x_{*}$ denoting the distance from the wavemaker. The disturbances at the leading edge of the truncated wave group are disregarded here. The growth of the maximum crest height with the distance is obvious, albeit nonmonotonic. In the uppermost curve in Fig. 4 the relative crest amplification exceeds the factor of 2 , as in Shemer and Liberzon (2014) at a comparable distance from the wavemaker. Here again, the broken line that corresponds to the propagation velocity of $c_{\mathrm{g}}$ clearly shows that the steepest crests in the train propagate at velocities exceeding $c_{\mathrm{g}}$.

A closer look at the surface elevation variation with time is presented in Fig. 5; the time here is shifted at each position by delay that would occur if the hump in the group indeed propagated with the group velocity. Actually, the maximum crest is invariably observed earlier. Both the vertical (trough- 


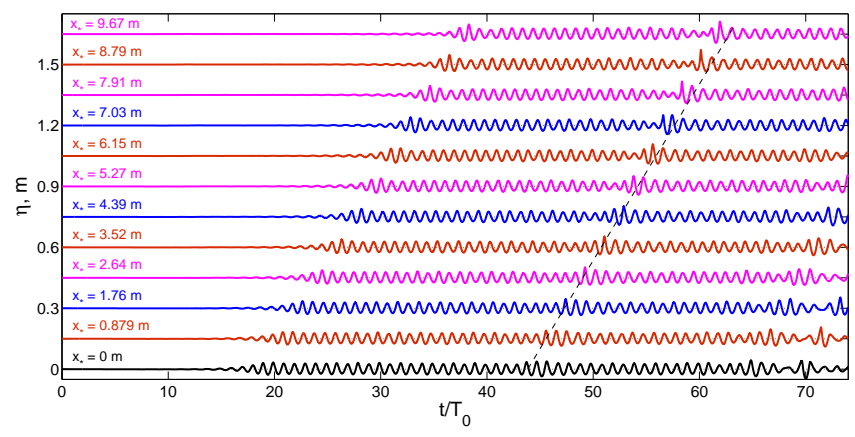

Figure 4. Surface elevation variation at fixed values of $x_{*}$ vs. time $t$. The group velocity is $c_{\mathrm{g}}=0.63 \mathrm{~m} \mathrm{~s}^{-1} ; x_{*}$ refers to the wave gauge distance from the wavemaker at $x_{*}=0$.

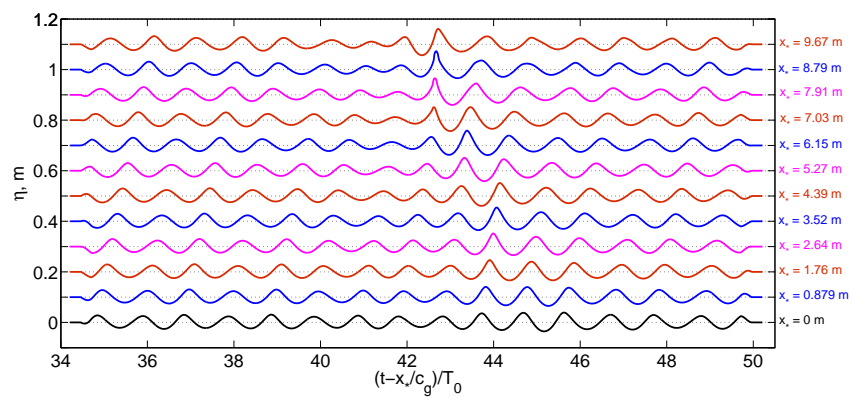

Figure 5. The computed temporal variation of the surface elevation at various locations relative to the wavemaker in a moving (with $c_{g}$ ) reference frame.

crest) and horizontal (right-left) asymmetries of steep waves are clearly visible in the plotted records.

In Fig. 6, we follow the highest crests in the instantaneous snapshots of wave trains. The elevations of the highest crests at each instant are compared with the propagation velocity of those crests, $v_{\mathrm{cr}}$. To enable comparison of parameters with different dimensions, crest heights are normalized by the background wave amplitude $\zeta_{0}$, while crest propagation velocities are normalized by the carrier wave phase velocity $c_{\mathrm{p}}$. Note that this figure corresponds to evolution times at which the hump's amplification is still relatively modest. Nevertheless, the crests propagate with time-dependent velocities $v_{\mathrm{cr}}$ that may be notably different from $c_{\mathrm{p}}$. It was demonstrated in experiments of Shemer and Liberzon (2014) that even for waves in the train that are far away from the hump and thus seem to be essentially monochromatic, the mean crest propagation velocity is somewhat higher than $c_{\mathrm{p}}$ due to the presence of the exponential term in Eq. (6).

It transpires from the comparison of the two curves in Fig. 6 that the higher the crests are, the lower their propagation velocity is. The minima in the instantaneous maximum crest heights correspond to the local maxima in their instantaneous propagation velocities. The averaged highest crest propagation velocity in Fig. 6 is $1.253 \mathrm{~m} \mathrm{~s}^{-1}$, slightly above $c_{\mathrm{p}}$.

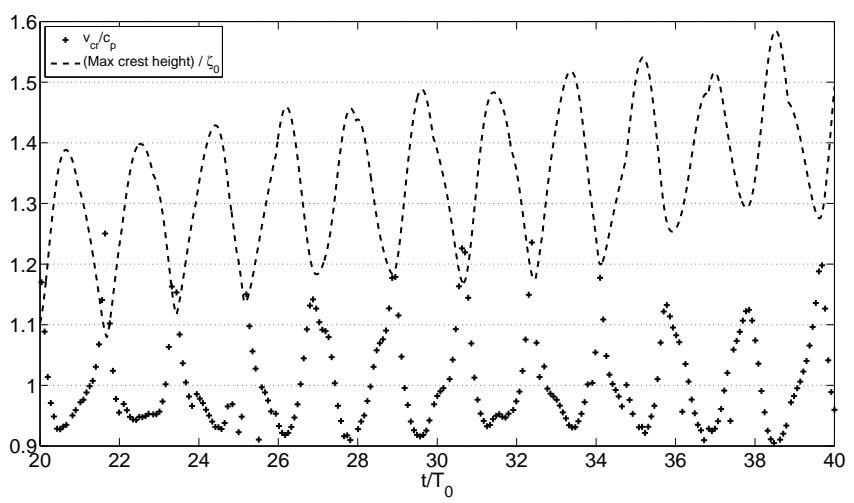

Figure 6. The computed variation with time of the normalized maximum crest elevation relative to the background wave amplitude $\zeta_{0}$ and the velocity of the highest crest propagation $\left(v_{\mathrm{cr}}\right)$ relative to the phase velocity $c_{\mathrm{p}}=1.248 \mathrm{~m} \mathrm{~s}^{-1}$.

The experiments were carried out with the goal of enabling quantitative comparison of the numerical results with experiments. The wavemaker driving signal was designed to generate surface elevation variation in time corresponding to the lowest curve in Fig. 4. Measurements were performed by multiple (up to four) resistance-type wave gauges placed on a bar in the center of the tank and connected to a computer-controlled carriage. The spacing between adjacent gauges was $0.4 \mathrm{~m}$. At each run, the position of the carriage was set by computer. Each successive run was initiated only after any disturbance of the water surface from the previous run had fully decayed. Measurements performed in different runs at fixed locations demonstrated excellent repeatability of results. Thus, the data collected at different locations obtained in various runs could be compared using the initiation of the wavemaker driving signal as a common temporal reference. Multiple experimental runs with different carriage positions provided experimental records of the temporal variation of the surface elevation in the wave train propagating along the whole tank with spacing that did not exceed $0.2 \mathrm{~m}$; denser measurements were carried out in the vicinity of the locations where inception of breaking was detected in visual observations.

An example of the sequence of the experimentally recorded wave trains for $6.6 \leq x \leq 7.8 \mathrm{~m}$ is presented in Fig. 7. Modifications of the wave train shape between the adjacent locations are relatively minor. The variation along the tank of the location and height of the steepest crest in the central part of the train can be easily followed from these records. Note that the highest crest at $x=7.6 \mathrm{~m}$ ceases to be such at $x=7.8 \mathrm{~m}$, where the following wave in the train becomes the steepest one. Such a transition of the highest crest in the train from one wave to another causes discontinuity in the velocity of propagation of the steepest crest; see e.g. Shemer and Liberzon (2014). 


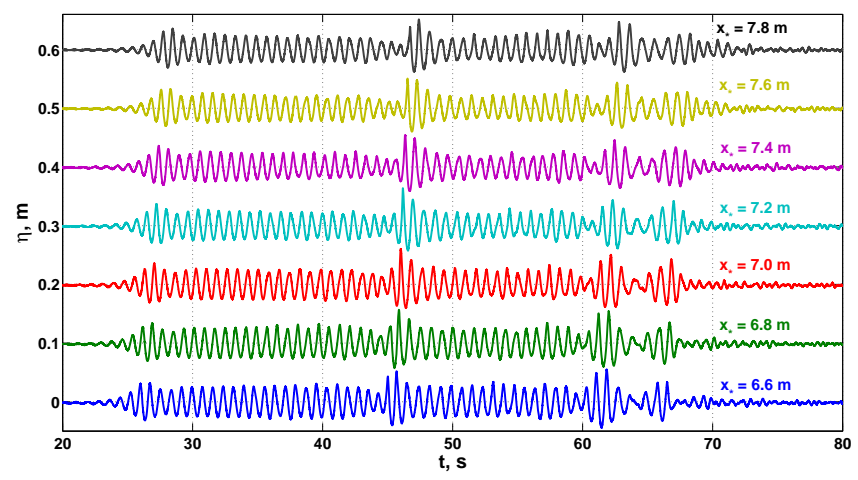

Figure 7. Measured surface elevation $\eta$ at various locations.
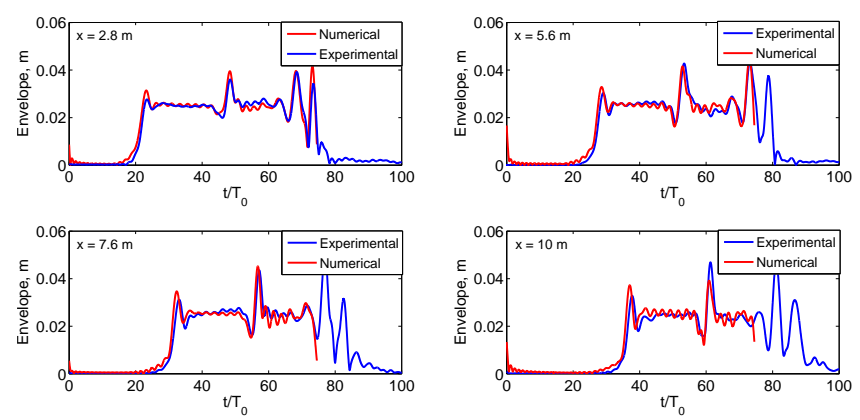

Figure 8. Comparison between the envelopes of the measured and numerical surface elevations $\eta$ at various locations.

It is impractical to carry out direct comparison of the fast varying surface elevation records measured in the experiments as presented in Fig. 7, with the corresponding numerical results. In order to compare the computed and measured results, the corresponding envelopes were computed; the absolute values of the measured and simulated wave train envelopes are presented in Fig. 8 for various distances from the wavemaker. To calculate the envelopes of the wave train in both simulations and experiments, the records were first band-pass filtered in the domain $0.5 \omega_{0} \leq \omega \leq 1.5 \omega_{0}$. This procedure leaves only the "free" waves, while the higherorder "bound" waves that cause vertical asymmetry of the records are removed. The envelopes of the filtered signals were then computed using the Hilbert transform. For more details, see e.g. Shemer et al. (1998).

Figure 8 demonstrates that essential similarity exists between the shapes of the measured wave trains at different distances from the wavemaker and those obtained in the numerical simulations. The propagation velocities of the leading edge of the wave train, as well as of the steepest crest, are also quite close in simulations and in experiments. The agreement between the numerical solution and the experimental results is, however, not perfect; the differences cannot be attributed to experimental errors only.

Important parameters of the wave train in the course of its propagation along the tank obtained in the simulations

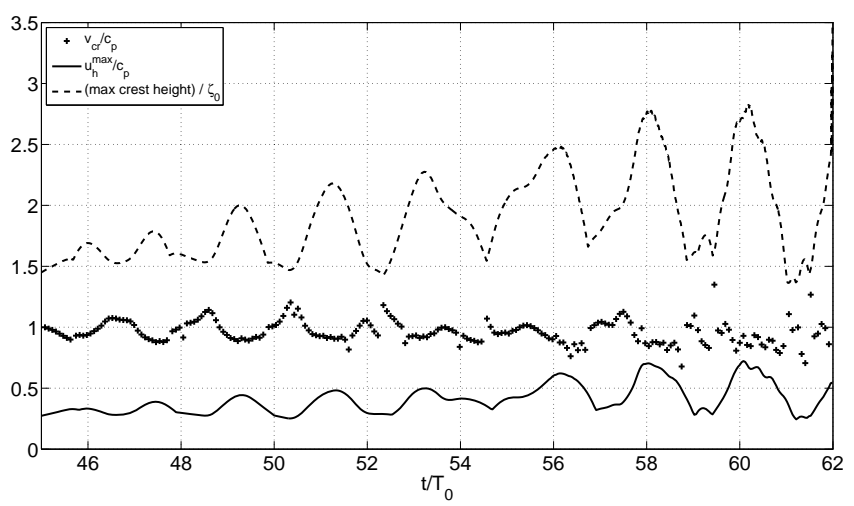

Figure 9. The computed temporal variation of the maximum crest heights, of the velocities of the steepest crests $\left(v_{\mathrm{cr}}\right)$, and of maximum horizontal fluid velocity $\left(u_{\mathrm{h}}^{\max }\right)$.

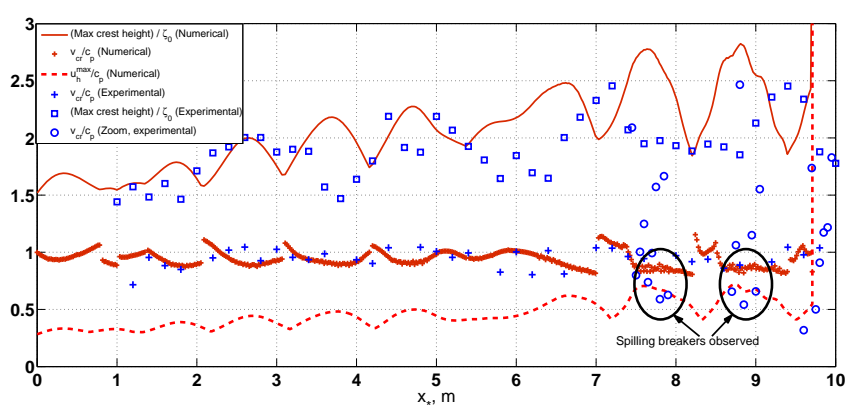

Figure 10. Variation along the tank of the maximum crest heights, propagation velocity of the highest crests, and the water particle velocity at those crests. Red lines denote numerical results, blue symbols experiments. The locations where breaking was observed in the experiments are marked.

are plotted in Figs. 9 and 10. In Fig. 9, the temporal variations of the computed velocities of the highest crests at each instant, $v_{\mathrm{cr}}$, and of the fluid velocity at those crests, $u_{\mathrm{h}}^{\max }$, are presented at late stages of the evolution, up to the apparent breakdown of computations at $t / T_{0} \approx 74$. However, the maximum crest height amplification exceeding 3 was observed at $t / T_{0} \approx 62$. The maximum crest elevations are also plotted in this figure for comparison. To enable comparison, all data are rendered dimensionless by normalizing them by their appropriate characteristic values. The fluid velocities increase with crest heights, while the crest propagation velocities decrease. At final stages the fluid velocity at the crest seems to exceed the crest velocity. The corresponding spatial variations are plotted in Fig. 10. In this figure, whenever available, the related experimental results are plotted as well.

The evolution of the steepest crest heights along the tank, as plotted in Fig. 10, in simulations and in experiments exhibit qualitative and to some extent quantitative similarity. The steepest crest heights have a tendency to grow along the tank; this growth is essentially non-monotonic in computations as well as in measurements. At distances from the 
wavemaker beyond $7 \mathrm{~m}$ the measured steepest crest heights may exceed the background by a factor of 2.5 ; the amplification factor in simulations is somewhat higher than that. The propagation velocity of the steepest crest, $v_{\mathrm{cr}}$, varies to a certain extent in experiments and in computations, remaining close to the phase velocity of the carrier wave, $c_{\mathrm{p}}$. The discontinuity in the steepest crest propagation velocity obtained in the experiments is related to the transition of the steepest crest in the train from one wave to another, as discussed with relation to Fig. 7. The spatial resolution of the determination of $v_{\mathrm{cr}}$ is obviously much better in the numerical simulations than in the experiments. For that reason, oscillations of the measured steepest crest velocity are less pronounced in the experimental results. As discussed with respect to the temporal variation of $c_{\mathrm{cr}}$ in Fig. 6, the crest propagation velocity decreases when crests become higher. This feature is more visible in the results of simulations as compared to the measurements due to their better resolution.

The bottom curve in Fig. 10 represents the variation along the tank of the instantaneous water particle velocity at the steepest crest, computed as $u_{\mathrm{h}}^{\max }=\partial \phi^{\mathrm{s}} / \partial x$ at the crest. This velocity varies in accordance with the variation of the crest height; as the crest becomes higher, the values of $u_{\mathrm{h}}^{\max }$ grow and may exceed notably the group velocity $c_{\mathrm{g}}$. Nevertheless, for the whole domain of computations, the horizontal liquid velocity at the crest remains lower than the computed $v_{\mathrm{cr}}$. Note that the computed temporal variations of $v_{\mathrm{cr}}$ and $u_{\mathrm{h}}^{\max }$ plotted in Fig. 9 demonstrate that the values of $v_{\text {cr }}$ may decrease below the local maximum of $u_{\mathrm{h}}^{\text {max }}$; however, this does not occur simultaneously. No measurements of $u_{\mathrm{h}}^{\max }$ were carried out in this study; however, detailed results on the Lagrangian kinematics at the wave crest approaching breaking obtained using particle tracking velocimetry were presented for the identical carrier wave parameters and somewhat different wavemaker driving signal in Shemer and Liberzon (2014).

At distances exceeding about $7 \mathrm{~m}$ from the wavemaker, the pattern of variation of the steepest crests' height and of their propagation velocity $v_{\text {cr }}$ plotted in Fig. 10 becomes less organized. In experiments, inception of the spilling breaker was observed at those distances; see the video in the Supplement. In order to obtain more accurate estimates of $v_{\text {cr }}$ in this region, measurements of the surface elevation were performed every $0.1 \mathrm{~m}$. The resulting steepest crest propagation velocities are plotted in Fig. 10 using different symbols. These results demonstrate that at the locations where the spilling breakers were observed, the measured $v_{\text {cr }}$ may indeed fall below the computed water surface velocity at the crest, $u_{\mathrm{h}}^{\max }$.

It was suggested in Shemer and Liberzon (2014) that spilling breakers appear when the horizontal water particle velocity at the steep crest $u_{\mathrm{h}}^{\max }$ attains the instantaneous crest propagation velocity $v_{\mathrm{cr}}$. While spilling breakers were clearly seen in the experiments at distances of about 7.5-8 and 8.5$9 \mathrm{~m}$ from the wavemaker (see the video in the Supplement), in computations the values of $u_{\mathrm{h}}^{\max }$, while increasing at steep

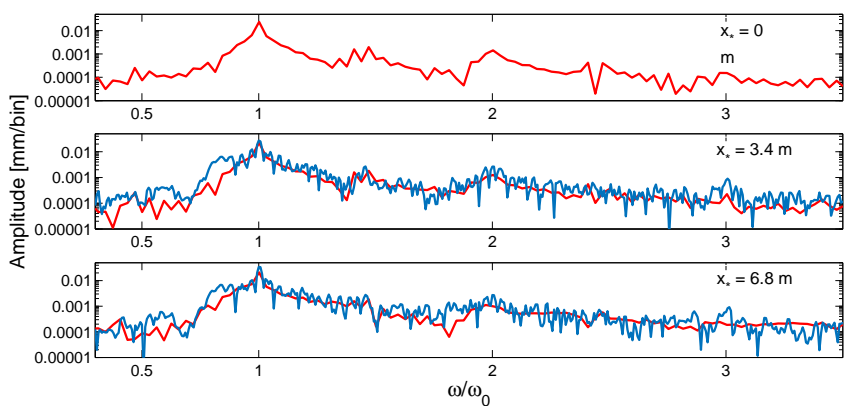

Figure 11. Frequency spectra at selected locations. Red line - numerical simulations; blue line - experimental data.

crests, remain consistently lower by about $10 \%$ than the computed $v_{\mathrm{cr}}$, although extremely low steepest crest propagation velocities were occasionally obtained numerically; see Fig. 9. The experimentally determined values of crest propagation velocity may indeed fall below the computed water particle velocity $u_{\mathrm{h}}^{\max }$.

In this respect it should be stressed that the velocities $u_{\mathrm{h}}^{\max }$ and $v_{\text {cr }}$ obtained in the present fully nonlinear numerical simulations, while apparently close to their actual values as demonstrated in Fig. 10, cannot be seen as the exact ones. Although special effort has been made in this study to calculate the required conditions at the wavemaker that correspond to the numerical solution, there remains a certain discrepancy between the computed and measured initial conditions due to an essentially nonlinear character of the wavemaker transfer function. While the differences are small, they can affect the exact locations of the observed extremal values of the surface elevation and the horizontal velocity. The experimental determination of $v_{\mathrm{cr}}$ as performed in the present study is inaccurate mainly due to the presence of unavoidable low-level noise in the surface elevation records that limit the precision of defining the instant when the maximum surface elevation is attained. The PTV-derived results on $u_{\mathrm{h}}^{\max }$ presented in Fig. 7 of Shemer and Liberzon (2014) show that horizontal surface velocities as high as $0.8 c_{\mathrm{p}}$, notably higher than the maximum values of $u_{\mathrm{h}}^{\max }$ in Fig. 10, were indeed measured at the breaking location. It should be noted that since the wavemaker is an essentially nonlinear device, it is difficult to adjust the actual surface elevation variation in the tank to that prescribed by the computations. It thus can be concluded that the differences between $u_{\mathrm{h}}^{\max }$ and $v_{\text {cr }}$ obtained numerically and those measured in the tank as presented in Fig. 10 stem from less than perfect matching between the initial conditions in the experiment and the numerical simulations. The total body of numerical and experimental results thus provides further support for the validity of the kinematic breaking criterion according to which the spilling breaker emerges when the instantaneous liquid velocity at the crest, $u_{\mathrm{h}}^{\max }$, attains that of the crest, $v_{\mathrm{cr}}$. 


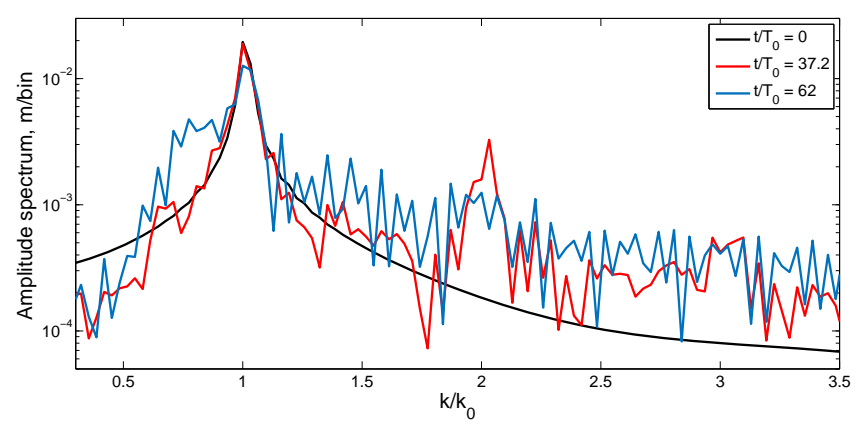

Figure 12. Wavenumber spectra at selected times.

The amplitude spectra of the wave train are plotted in Figs. 11 and 12. In Fig. 11, the numerically derived frequency spectra of $\eta(t)$ are compared at selected values of $x_{*}$ with the corresponding experimental results. At the wavemaker $\left(x_{*}=0\right)$, the spectrum in linear-logarithmic coordinates still retains resemblance to the triangular shape characteristic of the Peregrine breather. Nevertheless, a weak asymmetry around the dominant frequency $\omega_{0}$ can already be noticed at this location. Note that at $x_{*}=0$ the wave train already evolved over significant duration from its initial PB shape in Fig. 1. The non-negligible contribution of lowfrequency as well as second and third bound wave harmonics is also evident. The spectral asymmetry gets stronger and the spectrum widens with the distance from the wavemaker. Reasonable agreement is obtained between the experimental and numerical results. The wavenumber spectrum for the computed variation of $\eta(x)$ plotted in Fig. 12 at selected instants $t$ cannot be compared with the experiment. This is due to the fact that the spatial extent of the wave train exceeds significantly the length of the tank; see Fig. 2. Note that even for significantly shorter wave trains, the experimental procedure that enables extraction of wavenumber spectra (as opposed to frequency spectra) is extremely tedious; see Shemer and Dorfman (2008). The temporal evolution of wavenumber spectra in Fig. 12 is qualitatively similar to that discussed with respect to Fig. 11. The initial spectrum is nearly symmetric around the dominant wave number. Then, the spectrum widens with time and becomes more asymmetric towards breaking.

\section{Discussion and conclusions}

In the present study, fully nonlinear numerical simulations of the evolution of a unidirectional nonlinear wave train with an initial shape of a Peregrine breather were qualitatively and quantitatively compared with the experimental results. To the best of our knowledge, this is the first attempt to carry out direct comparison of the results of fully nonlinear simulations of a deterministic wave train with experiments. The simulations were carried out using a conformal mapping approach as detailed in Chalikov and Sheinin (2005). To validate the accuracy of the code, the computational results were reproduced using an alternative numerical approach of Milewski et al. (2010). These and some other numerical methods that are often applied to solve wave propagation problems require complete information on the wave field over the entire computational range at a certain instant. These initial conditions are unavailable in any controlled wave experiment in a laboratory facility. The unidirectional wave field in a tank is in fact prescribed by the wavemaker that is usually located at one end of the facility and driven by a computer-generated signal.

The initial condition in experiments thus corresponds to surface elevation variation with time at a prescribed wavemaker location. Shemer and Dorfman (2008) sought to reconcile the fundamental difference between the initial spatial distribution of wave field parameters required for the numerical solution, and the temporal variation of the surface elevation at the wavemaker prescribed as the initial condition in the experiments. This approach was generalized here to a fully nonlinear wave field with an arbitrary spectral width, thus enabling one to carry out consistent quantitative comparison of the results of numerical simulations and measurements.

In the present simulations, the initial spatial distribution of the surface elevation is based on the PB analytical solution. In order to determine in the numerical solution the measurable temporal variation of the surface elevation at any given location along the tank, the initial spatial distribution in the present study was centered upstream of the wavemaker; see Fig. 2. The appropriate location of the wavemaker was determined then by comparing the surface elevation variation in time with that measured in the experiment. The wavemaker driving signal generates surface elevation variation in time that corresponds to the bottom curve in Fig. 4. This dependence that is very different from the analytical solution given by PB is obtained as a result of the evolution of the wave train with an initial shape given in the bottom panel of Fig. 1. It enables detailed and quantitative comparison of the simulations with experiment.

Several important points regarding PB were highlighted in this study. The solution (Eq. 6) of the spatial form of the nonlinear Schrödinger equation (Eq. 2) is aperiodic in space due to the presence of an exponential term. Similarly, the temporal form of the NLS equation (Lo and Mei, 1985; Shemer and Dorfman, 2008) yields PB that has an asymmetry in time. The exact shape of the analytical solution (Eq. 6) thus cannot be reproduced either in the experiments or in computations. Note that in the present study, the actual initial condition for the simulations and the wavemaker driving signal have been modified and are fundamentally different from PB. The presented results on steep crests in the wave train are therefore of a generic nature and applicable beyond the 2-D PB wave packets.

Two different approaches were suggested to deal with the problems outlined in the previous paragraph. In numerical 
simulations, the truncated wave train with the spatial extension that contains an integer number of carrier wavelengths is often used as the initial condition (Slunyaev and Shrira, 2013). However, imposing a non-zero periodic boundary condition on an essentially aperiodic function may affect significantly the nature of the solution. It was thus decided in the present study to follow the experimental approach of Shemer and Alperovich (2013) and Shemer and Liberzon (2014). The theoretical solution given by Eq. (6) was truncated and tapered before being used to determine the initial condition at the wavemaker. In order to mitigate the effect of truncation on the central part of the wave train, a sufficiently large number of wave periods (about 70) was used in those experiments. A similar approach was adopted in the present study. As can be seen in both experimental and numerical results (Figs. 1, 4 and 9), truncation and tapering, while indeed satisfying periodic boundary conditions in the computational domain, cause appearance of abnormally high waves at the leading and trailing edges of the wave train. The effect of truncation is apparently limited to the edges of the train, and does not affect the behavior of the central part of the PB-like wave train with the gradually amplified, albeit non-monotonically, hump in the envelope. These high waves do not characterize the wave train proper and therefore were disregarded in the present study.

The computational results indeed are in a good qualitative and, to a large extent, quantitative agreement with the current experiments, as well as with those of Shemer and Liberzon (2014). This includes the behavior of the truncated train edges, the amplification of the hump along the tank, the asymmetric spectral widening, as well as the variation of the envelope shape along the tank. Crest slowdown was noted by Johannssen and Swan in fully nonlinear calculations (Johannessen and Swan, 2001) and experiments (Johannessen and Swan , 2003). The slowdown of crests in the PB-like wave train as they grow in height was first observed by Shemer and Liberzon (2014) in experiments and NLS solutions and is of particular interest. More recently, this effect was also stressed in the context of focusing of 2-D and 3-D nonlinear deep water wave packets by Banner et al. (2014), and for 2-D nonlinear wave packets by Kurnia and Groesen (2014) as well as by Fedele (2014), thus providing additional evidence of the generic nature of the phenomenon. It was noticed in Shemer and Liberzon (2014) that the increase in the maximum crest height along the tank is not monotonic. As the maximum crest height increases, the water particles at the crest accelerate to higher maximum velocities $u_{\mathrm{h}}^{\max }$, while the crest propagation speed $v_{\mathrm{cr}}$ decreases. The equality $u_{\mathrm{h}}^{\max }=v_{\mathrm{cr}}$ was thus suggested as the kinematic criterion for wave breaking. A slightly different version of this criterion was offered by Kurnia and Groesen (2014); they maintain that the maximum liquid particle velocity $u_{\mathrm{h}}^{\max }$ exceeds about $0.8 v_{\text {cr }}$ at breaking. If only the simulations are considered, it seems that this somewhat weaker version of the kinematic breaking criterion is confirmed. However, the present experimental as well as numerical results, combined with those obtained experimentally by alternative methods in Shemer and Liberzon (2014), provide strong, albeit not fully conclusive, support for the conjecture that indeed the particle velocities at the inception of breaking attain and exceed the crest propagation velocities and thus for the kinematic breaking criterion in the formulation suggested in that study. This conjecture is further corroborated by visual evidence as seen in video clips presented in Supplements to Shemer and Liberzon (2014) and to the present study. ${ }^{1}$

This combined numerical and experimental study of nonlinear wave trains also clarifies the limitations of possible agreement between fully nonlinear solution and experiment. We note that while the periodicity in the time domain is possible for propagating and evolving unidirectional waves, they are, strictly speaking, aperiodic in space. This point adds an additional aspect to essential differences that exist between the spatial and temporal formulations of the wave evolution problem, as discussed above. We therefore believe that all nonlinear solutions based on spatially periodic boundary conditions, as in the method adopted here, as well as in a variety of alternative methods that employ spatial discrete Fourier decomposition, contain intrinsic inaccuracy. These numerical solutions thus can only provide approximate results and require careful experiments to verify their validity. The present study shows that the fully nonlinear solution, although flawed, yields better agreement with experiments than the application of the spatial version of the modified nonlinear Schrödinger (Dysthe) equation limited to the third order that does not require spatial periodicity (Shemer and Alperovich (2013).

\section{The Supplement related to this article is available online at doi:10.5194/npg-22-737-2015-supplement.}

Acknowledgements. The support of this study by a grant no. 20102019 from the US-Israel Binational Science Foundation is gratefully acknowledged. The authors wish to thank Andrey Zavadsky for his valuable assistance.

Edited by: V. Shrira

Reviewed by: two anonymous referees

\section{References}

Akhmediev, N. N., Eleonskii, V. M., and Kulagin, N. E.: Exact firstorder solutions of the nonlinear Schrödinger equation, Theor. Math. Phys., 72, 809-818, 1987.

\footnotetext{
${ }^{1}$ A different approach was suggested by Saket et al., 2015, ArXiv 1508.07702, and Barthelemy et al., 2015, ArXiv 1508.06002, after this manuscript was submitted.
} 
Babanin, A., Chalikov, D., Young, I., and Savelyev, I.: Predicting the breaking onset of surface water waves, Geophys. Res. Lett., 34, L07605, doi:10.1029/2006GL029135, 2007.

Banner, M. L., Barthelemy, X., Fedele, F., Allis, M., Benetazzo, A., Dias, F., and Peirson, W. L.: Linking reduced breaking crest speeds to unsteady nonlinear water wave group behavior, Phys. Rev. Lett., 112, 114502, doi:10.1103/PhysRevLett.112.114502, 2014.

Bateman, W. J. D., Kasardi, V., and Swan, C.: Extreme ocean waves. Part I. The practical application of fully nonlinear wave modeling, Appl. Ocean. Res., 34, 209-224, 2012.

Chabchoub, A., Hoffmann, N. P., and Akhmediev, N.: Rogue wave observation in a water wave tank, Phys. Rev. Lett. 106, 204502, 2011.

Chabchoub, A. S., Neumann, S., Hoffmann, N. P., Akhmediev, N.: Spectral properties of the Peregrine soliton observed in a water wave tank. J. Geophys. Res. 117, C00J03, doi:10.1029/2011JC007671, 2012

Chalikov, D. and Sheinin, D.: Direct modeling of one-dimensional nonlinear potential waves, Nonlinear Ocean Waves, edited by: Perrie, W., Adv. Fluid Mech. Ser., 17, 207-258, 1998.

Chalikov, D. and Sheinin, D.: Modeling of extreme waves based on equations of potential flow with a free surface, J. Comput. Phys., 210, 247-273, 2005.

Dysthe, K. B.: Note on the modification of the nonlinear Schrödinger equation for application to deep water waves, P. Roy. Soc. Lon. A Mat., 369, 105-114, 1979.

Dysthe, K. B. and Trulsen, K.: Note on breather type solutions of the NLS as models for freak-waves, Phys. Scripta, 82, 48, doi:10.1238/Physica.Topical.082a00048, 1999.

Dysthe, K., Krogstad, H. E., and Müller, P.: Oceanic rogue waves, Annu. Rev. Fluid Mech., 40, 287-310, 2008.

Fedele, F.: Geometric phases of water waves, EPL, 107, 69001, doi:10.1209/0295-5075/107/69001, 2014.

Hasimoto, H. and Ono, H.: Nonlinear modulation of gravity waves, J. Phys. Soc. Jpn., 33, 805-811, 1972.

Johannessen, T. B. and Swan, C.: A laboratory study of the focusing of transient and directionally spread surface water waves, Proc. R. Soc. A, 457, 971-1006, 2001.

Johannessen, T. B. and Swan, C.: On the nonlinear dynamics of wave groups produced by the focusing of surface-water waves, Proc. R. Soc. A, 459, 1021-1052, 2003.

Kharif, C., Pelinovsky, E., and Slunyaev, A.: Rogue Waves in the Ocean, Springer Verlag, Germany, 216 pp., 2009.

Kit, E. and Shemer, L.: Spatial versions of the Zakharov and Dysthe evolution equations for deep water gravity waves, J. Fluid Mech., 450, 201-205, 2002.

Kurnia, R. and Groesen, E. V.: High order Hamiltonian water wave models with wave-breaking mechanism, Coast. Eng., 93, 55-70, 2014.

Kuznetsov, E. A.: Solitons in a parametrically unstable plasma, Akad. Nauk SSSR Dokl., 236, 575-577, 1977.

Lo, E. and Mei, C. C.: A numerical study of water-wave modulation based on higher-order nonlinear Schrödinger equation, J. Fluid Mech., 150, 395-416, 1985.

Ma, Y. C.: The perturbed plane-wave solutions of the cubic Schrödinger equation, Stud. Appl. Math., 60, 43-58, 1979.

Mei, C. C.: The Applied Dynamics of Ocean Surface Waves, World Scientific, Singapore, 740 pp., 1989.
Milewski, P. A., Vanden-Broeck, J. M., and Wang, Z.: Dynamics of steep two-dimensional gravity-capillary solitary waves, J. Fluid Mech., 664, 466-477, 2010.

Osborne, A., Onorato, M., and Serio, M.: The nonlinear dynamics of rogue waves and holes in deep-water gravity wave train, Phys. Lett. A, 275, 386-393, 2000.

Peregrine, D. H.: Water waves, nonlinear Schrödinger equations and their solutions, J. Aust. Math. Soc. B, 25, 16-43, 1983.

Perić, R., Hoffmann, N., and Chabchoub, A.: Initial wave breaking dynamics of Peregrine-type rogue waves: a numerical and experimental study, Eur. J. Mech. B-Fluids, 49, 71-76, 2014.

Phillips, O. M.: The equilibrium range in the spectrum of wind generated waves, J. Fluid Mech., 4, 426-434, 1958.

Shemer, L.: On kinematics of very steep waves, Nat. Hazards Earth Syst. Sci., 13, 2101-2107, doi:10.5194/nhess-13-21012013, 2013.

Shemer, L.: The advantages and limitations of the nonlinear Schrödinger equation in description of evolution of nonlinear water-wave groups. Proc. Estonian Acad. Sci. 64, 3S, 356-360, doi:10.3176/proc.2015.3S.05, 2015.

Shemer, L.: Quantitative Analysis of nonlinear water-waves: a perspective of an experimentalist, New Approaches to Nonlinear Waves, edited by: Tobisch, E., Lecture Notes in Physics, 908, 211-293, Springer, Switzerland, 2016.

Shemer, L. and Alperovich, L.: Peregrine Breather revisited, Phys. Fluids, 25, 051701, doi:10.1063/1.4807055, 2013.

Shemer, L. and Dorfman, B.: Experimental and numerical study of spatial and temporal evolution of nonlinear wave groups, Nonlin. Processes Geophys., 15, 931-942, doi:10.5194/npg-15-9312008, 2008.

Shemer, L. and Liberzon, D.: Lagrangian kinematics of steep waves up to the inception of a spilling breaker, Phys. Fluids, 26, 016601, doi:10.1063/1.4860235, 2014.

Shemer, L., Kit, E., Jiao, H., and Eitan, O.: Experiments on nonlinear wave groups in intermediate water depth, J. Waterw. Port C.-ASCE, 124, 320-327, 1998.

Shemer, L., Jiao, H. Y., Kit, E., and Agnon, Y.: Evolution of a nonlinear wave field along a tank: experiments and numerical simulations based on the spatial Zakharov equation, J. Fluid Mech., 427, 107-129, 2001.

Shemer, L., Kit, E., and Jiao, H. Y.: An experimental and numerical study of the spatial evolution of unidirectional nonlinear waterwave groups, Phys. Fluids, 14, 3380-3390, 2002.

Shemer, L., Goulitski, K., and Kit, E.: Evolution of wide-spectrum wave groups in a tank: an experimental and numerical study, Eur. J. Mech. B-Fluid., 26, 193-219, 2007.

Shrira, V. I. and Geogjaev, V. V.: What makes the Peregrine soliton so special as a prototype of freak waves?, J. Eng. Math., 67, 1122,2010

Slunyaev, A. V. and Shrira, V. I.: On the highest non-breaking wave in a group: fully nonlinear water wave breathers versus weakly nonlinear theory, J. Fluid Mech., 735, 203-248, 2013.

Zakharov, V. E.: Stability of periodic waves of finite amplitude on a surface of deep fluid, J. Appl. Mech. Tech. Phys., 9, 190-194, 1968. 